\title{
XLIII. The calculation of the virtual resistance of thin wires for rapidly alternating currents
}

\section{Professor A. Gray F.R.S.}

To cite this article: Professor A. Gray F.R.S. (1898) XLIII. The calculation of the virtual resistance of thin wires for rapidly alternating currents , Philosophical Magazine Series 5, 46:281, 426-428, DOI: $10.1080 / 14786449808621213$

To link to this article: http://dx.doi.org/10.1080/14786449808621213

$$
\text { Published online: } 08 \text { May } 2009 .
$$

\section{Submit your article to this journal $\square$}

Џ Article views: 2 
Thus the statement No. 5, made above, is incomplete and inaccurate without the proviso about a steady state of the æther. It appears to be true, so long as it is a case of the statical exertion of force, that such force can only be exerted by matter; but it appears also to be true that the ather or any other medium capable of transmitting rays is able to experience a force on the boundary between two regions possessing different intensities of vibrational energy (the force per unit urea being equal to the difference in the energies per unit volume), provided that this boundary advances at a certain speed appropriate to the medium, viz. the speed at which it transmits that particular kind of vibrational energy, or provided that it retreats at the same rate in a direction opposed to the force,-in this latter case destroying the wave-energy which previously existed in the medium.

Hence 5 may be generalized thus :-

5'. A stress occurs either in the space between two material bodies, or between a body and an advancing wave-front, or between an advancing and a retreating wave-front. Its action and reaction are always equal and opposite, and a free ctherial reaction is necessarily transmitted with the speed of light.

In other words, a stress whose one end terminates in æether is necessarily a growing or decaying (or, in the case of both ends, a sbifting) and not a statical stress; it is doing work at a certain definite rate, and thereby generating or destroying ætherial wave-energy. The mechanical force acts not only at obvious wave-fronts, but at every boundary on one side of which there is electric accoleration, $i$. $e$. where electric and magnetic forces coexist; and its value is $\mathrm{V}(\mathrm{EH})$. Thus a boundary between pulsating and inert æther behaves in some respects like a material partition; it is able on certain conditions to take the place of a reacting body in Newton's third law. This is suggestive in comnexion with the view that regards all matter as a variety of wtherial strain and motion. The normal pressure on such a boundary results not in normal but in tangential (electric) yield; there is probably very little meaning in disentangling cause and effect, otherwise the gyrostatic analogy is suggestive.

XLIII. The Calculation of the Virtual Resistance of Thin Wires for Rapidly Alternating Currents. By Professor A. GRAT, F.R.S.

To the Editors of the Philosoplical Magazine. GenTlemen,

I $\mathrm{N}$ his interesting paper on "The High-Frequency Induc1 tion-Coil," published in the current number of the Philosophical Magazine, Mr. W. P. Boynton quotes an expression, 
given by $\mathbf{M r}$. Mathews and myself in our treatise on Bessel Functions, for the resistance of a straight metallic wire carrying a very rapidly alternating current. The equation we give is

$$
\mathrm{R}^{i}=\sqrt{\frac{\mu n l \mathrm{R}}{2}}
$$

where $\mathbf{R}$ is the resistance of a wire of length $l$ and permeability $\mu$ to steady currents, and $\mathrm{R}^{\prime}$ is its virtual resistance to currents of frequency $n / 2 \pi$. Taking $\mu$ as unity, $r$ as the radius of the wire, and $k$ as its conductivity, Mr. Boynton transforms this equation to

$$
\mathrm{R}^{\prime}=\mathrm{R} r \sqrt{\overline{\pi n}}
$$

which leads to the result that for $n=500,000$ and $k=\cdot 0006$,

$$
\mathrm{R}^{\prime}=36,000 r \mathrm{R} \text {. }
$$

This result, as $\mathrm{Mr}$. Boynton says, is startling, and is not confirmed by his experiments.

I desire to point out that the transformed equation obtained by Mr. Boynton is not correct, and should stand

$$
\mathrm{R}^{\prime}=\mathrm{R} r \sqrt{\frac{\pi n k}{2}}
$$

which, with $n$ and $k$ as stated, gives

$$
\mathrm{R}^{\prime}=21 \cdot 7 r \mathrm{R} \text {. }
$$

For wire of 1 millimetre diameter-the case considered by Mr. Boynton, this becomes

$$
\mathrm{R}^{\prime}=1 \cdot 085 \mathrm{R},
$$

so that the virtual increase of resistance in this case works out to only $8.5 \mathrm{per}$ cent. This number, however, is not a close approximation to the true value of $R^{\prime}$. Obviously, if we were to make the radius of the wire about 8 per cent. less than half a millimetre, $21 \cdot 7 r \mathrm{R}$ would become $\mathrm{R}$, and further diminution of $r$ would give $\mathrm{R}^{\prime}<\mathrm{R}$, results which are clearly erroneous, To find a fair approximation recourse must be had to the series by which, for a given $n$ and a given $r$, the virtual resistance can be calculated. Thus I obtain, from three terms of the series, $R^{\prime}=1 \cdot 291 R$, which, as the next term is positive, must be somewhat too small.

The series is really one of ascending powers of the product $\mu^{2} n^{2} r^{4} k^{2}$; and it is only when this quantity is increased without limit that we obtain the limiting formula stated above. In other words, the formula gives the value of $\mathrm{R}^{\prime}$ for a given wire to a given degree of approximation only when $n$ is made sufficiently great; and it cannot be applied to a case in which, 
while $n$ is fixed at the fairly high value of $500,000, r$ is taken so small as half a millimetre.

I wish to add that the value of $R^{\prime}$ for alternating currents of any periodicity is really due to Lord Rayleigh, who first fully worked out this question. This acknowledgment, I find to-day on looking up the passage referred to by Mr. Boynton, we have inadvertently omitted. The particular Bessel Function method adopted in the book for obtaining the formulæ is the only thing to which we have any claim.

I remain, Gentlemen,

University College of N. Wales, Your obedient Servant, Bangor, Sept. 1, 1890.

ANDREW Gray.

XLIV. Intelligence and Miscellaneous Articles.

A NEW COMBINATION OF WHEEL-GEARING.

BY J. J. TAUDIN CHABOT.

IF we try to construct a skew-gearing (endless screw) with a 1 velocity-ratio of $1: 1$, we find that the two wheels become identical. These, repeated to any desired extent, are the elements of the model to be here discussed. The structure of the model may be described as follows:-The axes of rotation of the wheels coincide with the $x, y, z$ and $\phi, \chi, \psi$ axes of two equidistant systems of rectangular coordinates projecting into each other; on two of the axes of each of the two systems of coordinates there occur alternately a wheel and a zero-point (point of intersection with the other two axes of the same system); while on the third axis of each system there occur in order a wheel, a zero-point, a space without a wheel, a zero-point, a wheel, and so on in endless repetition. These two last-mentioned axes cross each other at right angles.

\section{Properties of the Molel.}

I. When all the wheels rotate gearing into each other, a negative or positive acceleration of the rotation of one of them causes the rotatory motion-whether of this wheel only or of the wheels directly or even indirectly in gear with it--to be partially transformed into a motion of translation. The wheel moves in one direction or the other along its axis of rotation until the acceleration ceases, or is replaced by one of opposite sign. In the former case the wheel continues to rotate in its new position; in the latter it returns by a reversed motion of translation to its original place (or goes beyond it, as the case may be).

II. If translatory motion is continuously maintained, the wheel may pass out of gear with the adjacent wheels, and may even gear in with an independent set of wheels.

III. The disturbances described under heads I. and II. extend uniformly in all directions throughont a sphere of finite dimensions. The working (regular rotation) of the wheels which at any instant lie outside the sphere of disturbance remains unaffected, even if they belong to the same model.-Communicated by the Author. 